\title{
The emerging role of hepatitis B virus Pre-S2 deletion mutant proteins in HBV tumorigenesis
}

\author{
Ih-Jen Su ${ }^{1,2^{*}}$, Lily Hui-Ching Wang ${ }^{4}$, Wen-Chuan Hsieh ${ }^{1}$, Han-Chieh Wu ${ }^{1}$, Chiao-Fang Teng ${ }^{1}$, Hung-Wen Tsai ${ }^{2}$ and \\ Wenya Huang ${ }^{3}$
}

\begin{abstract}
Chronic hepatitis B virus (HBV) infection can cause hepatocellular carcinoma (HCC). Several hypotheses have been proposed to explain the mechanisms of HBV tumorigenesis, including inflammation and liver regeneration associated with cytotoxic immune injuries and transcriptional activators of mutant HBV gene products. The mutant viral oncoprotein-driven tumorigenesis is prevailed at the advanced stage or anti-HBe-positive phase of chronic HBV infection. Besides HBX, the pre-S2 (deletion) mutant protein represents a newly recognized oncoprotein that is accumulated in the endoplasmic reticulum (ER) and manifests as type II ground glass hepatocytes (GGH). The retention of pre-S2 mutant protein in ER can induce ER stress and initiate an ER stress-dependent VEGF/Akt/mTOR and NFkB/ COX-2 signal pathway. Additionally, the pre-S2 mutant large surface protein can induce an ER stress-independent pathway to transactivate JAB-1/p27/RB/cyclin A,D pathway, leading to growth advantage of type II GGH. The pre-S2 mutant protein-induced ER stress can also cause DNA damage, centrosome overduplication, and genomic instability. In 5-10\% of type II GGHs, there is co-expression of pre-S2 mutant protein and HBx antigen which exhibited enhanced oncogenic effects in transgenic mice. The mTOR signal cascade is consistently activated throughout the course of pre-S2 mutant transgenic livers and in human HCC tissues, leading to metabolic disorders and HCC tumorigenesis. Clinically, the presence of pre-\$2 deletion mutants in sera frequently develop resistance to nucleoside analogues anti-virals and predict HCC development. The pre-S2 deletion mutants and type II GGHs therefore represent novel biomarkers of HBV-related HCCs. A versatile DNA array chip has been developed to detect pre-S2 mutants in serum. Overall, the presence of pre-S2 mutants in serum has implications for anti-viral treatment and can predict HCC development. Targeting at pre-S2 mutant protein-induced, ER stress-dependent, mTOR signal cascade and metabolic disorders may offer potential strategy for chemoprevention or therapy in high risk chronic HBV carriers.
\end{abstract}

Keywords: Ground glass hepatocytes, Pre-S mutants, Endoplasmic reticulum stress, Chronic HBV infection, Hepatocellular carcinoma

\section{Introduction}

Hepatitis B virus ( HBV ) has been well established to cause hepatocellular carcinoma ( HCCs ) [1]. Several hypotheses have been proposed to explain the mechanism of $\mathrm{HBV}$ tumorigenesis, including inflammation and liver regeneration associated with cytotoxic immune injuries, HBV DNA insertional mutagenesis, and viral oncoproteins-driven tumorigenesis [2-4]. Although $\mathrm{HCC}$ can occur at any stage of chronic HBV infection, the majority of cases occur at the

\footnotetext{
* Correspondence: suihjen@nhri.org.tw

${ }^{1}$ National Institute of Infectious Diseases and Vaccinology, National Health

Research Institutes, Tainan, Taiwan

${ }^{2}$ Department of Pathology, College of Medicine, National Cheng Kung

University, Tainan, Taiwan

Full list of author information is available at the end of the article
}

advanced stage or anti-HBe-positive phase with the peak incidence at sixth decade [4]. The development of HCC related to the inflammation and liver regeneration is likely due to the cytotoxic $\mathrm{T}$ cell immune injuries toward HBV antigen-expressing hepatocytes which may result in bridging hepatic necrosis and fibrosis [3,5]. In the anti-HBepositive phase, however, the mutant viral oncoproteins may play an important or driving role in HCC development.

In the early 1970s when the Australia antigen was found to be associated with hepatitis B virus (HBV) infection, Hadziyannis and Popper first recognized the surface antigen in the "ground glass" hepatocytes (GGH) of HBV carriers [6,7]. Under electron microscopy, GGHs are characterized by an abundance of endoplasmic reticulum (ER), among 
which particles of surface antigens accumulate [8]. It is believed that the overloaded ER makes the cytoplasms of GGHs become "foggy" or "glassy". After the introduction of immunohistochemistry, a series of studies demonstrated that different types of GGHs correlated to the expression patterns of HBV surface/core antigens and the replicative stages of chronic HBV infection $[9,10]$. Two types of GGHs were later designated by us as type I and II GGHs [11]. Type I GGHs are usually scattered singly in the hepatic lobules with the expression of "inclusion-like" pattern of surface antigens (Figure 1A). This type of GGHs usually occurs at the early stage or in patients with active diseases, frequently co-expressed with a nuclear or cytoplasmic core antigen $[9,12,13]$. Type II GGHs, however, express a unique expression pattern of surface antigens at the cell margin (Figure 1A). Most interestingly and importantly, type II GGHs consistently cluster in nodules and usually occur at the advanced stages or anti-HBe-positive phase [14], and are frequently associated with cirrhosis or hepatocellular carcinoma (HCC). Conversion from type I GGH to type II GGH could be demonstrated in the serial biopsies from the same individual, frequently associated with hepatitis B e antigen (HBeAg) seroconversion [14]. The consistent clustering distribution of type II GGHs, especially in the non-tumorous liver tissues of patients with HCC receiving surgery, drives us to hypothesize that type II GGHs may represent clonally-proliferated or preneoplastic lesions of HCC [15]. The distinct marginal expression pattern of surface antigens in type II GGHs suggests that there may exist a unique form of mutant surface proteins which exhibit growth advantage to promote the clustering distribution of hepatocytes.

The above-mentioned observation of the unique biologic and pathologic features of type II GGHs drives us to explore in details the underlying molecular and biologic mechanism of type II GGHs and its potential significance in HBV tumorigenesis. In the past decade, we clarified the biologic and molecular significance of type II GGHs which

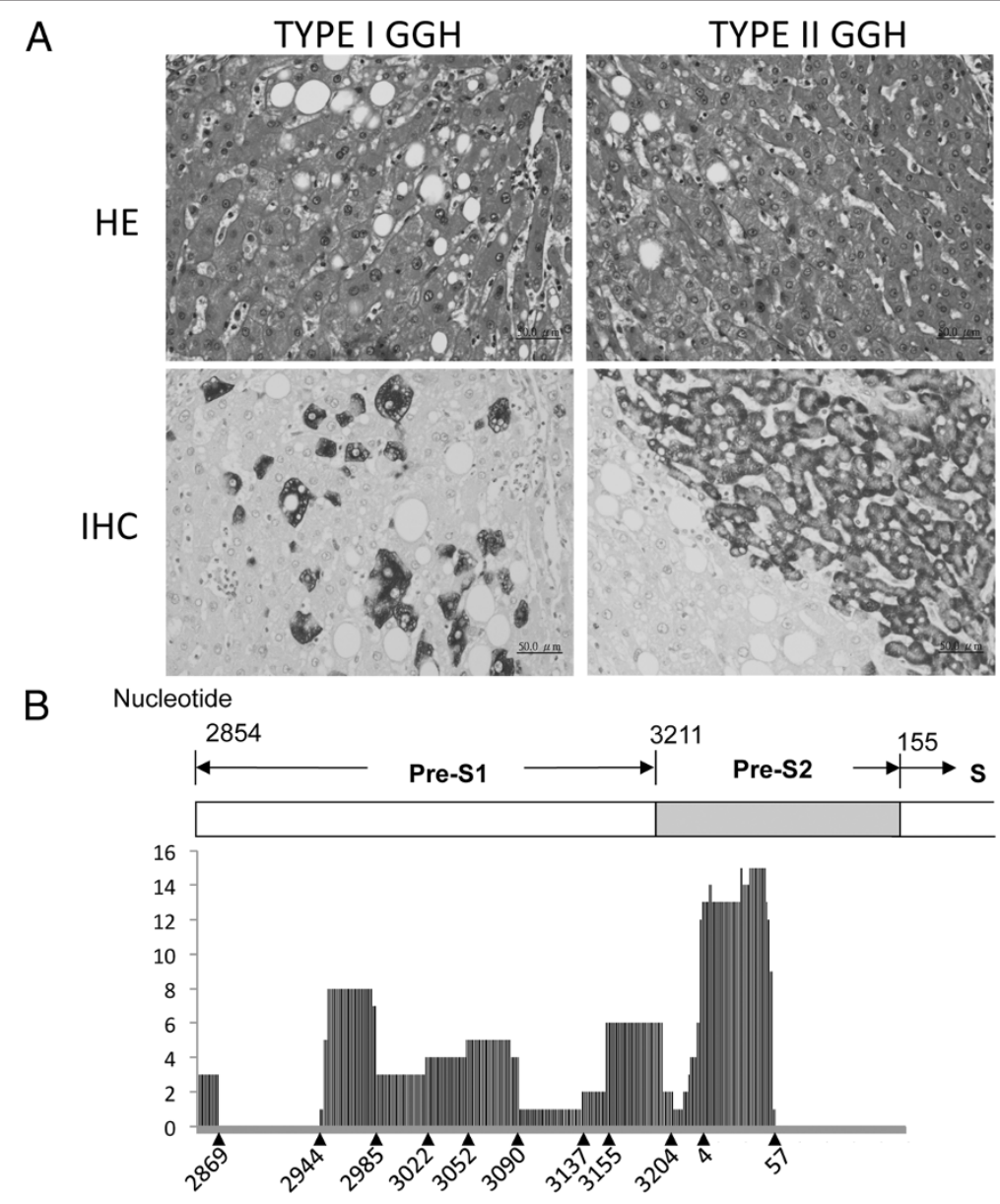

Figure 1 Two types of ground glass hepatocytes and the corresponding deletions at pre-S regions of large surface protein. Ground glass hepatocytes (GGH) and the expression patterns of hepatitis B surface antigens. Type I GGH are usually scattered singly and express an inclusion-like pattern of the surface antigen, while type II GGH consistently cluster in nodules and express a marginal pattern of the surface antigen (A). The profile of deletions over the pre-S regions in sera of HBV-related HCC patients. A complex combination of deletions at pre-S1 and pre-S2 regions may occur in patients of chronic HBV carriers (B). Hematoxylin-Eosin stain, HE; Immunohistochemical stain, IHC. 
contain a unique form of pre-S2 deletion mutant large surface protein (pre-S2 mutant). The significance of pre-S2 mutant proteins in HCC development, the signal pathway initiated by pre-S2 mutant proteins, and the transgenic mice model of pre-S2 mutant tumorigenesis were studied in detail. Importantly, we developed a DNA array chip to detect pre-S2 mutant gene as the predictive marker of HCC in chronic HBV carriers. Potential chemoprevention or therapy targeting at mTOR signal cascade and metabolic pathway was proposed in this review.

\section{Review}

Ground glass hepatocytes contain pre-S deletion mutant proteins which accumulate in endoplasmic reticulum (ER) and induce ER stress signals

By dissecting the cirrhotic nodules containing type II GGHs, we are surprised to find that type II GGHs consistently harbored pre-S2 mutants with in-frame deletions over the pre-S2 regions (predominantly, nt. 2-55 or 4-57) with or without point mutations at the start codon (ATG-ATA) of the middle surface gene in the pre-S2 region. These mutations lead to a decreased synthesis of middle and small surface antigens and result in a defective secretion of the mutant large surface antigens which then accumulate in $\mathrm{ER}$, leading to the GGH formation in chronic HBV infection $[11,15]$. It is also interesting to note that the deletion site at the pre-S2 region coincides with the epitope of cytotoxic T-cell and B-cell neutralization responses and suggests that the pre-S2 deletion mutants represent an immune escape mutant $[16,17]$, This hypothesis is supported by the pathologic observation that the hepatic lobules, which contain type II GGH, usually show no inflammatory activities or lymphocyte infiltration $[6,14,18]$. Distinct from type II GGHs, the singly distributed type I GGHs contain entirely different pre-S mutants with variable deletions over the pre-S1 regions. The deletion sites at the pre-S1 region may interfere with the transcriptional activities of the preS2/S promoter and affect the regulation of HBV replication and synthesis of small surface antigens $[19,20]$. The pre-S1 containing large surface antigen (LHB) exhibits a dual topology, and only half of the LHB translocate posttranslationally into the ER lumen [21,22]. The pre-S regions that remain in cytoplasmic orientation can bind to the cytosolic heat shock protein Hsc70 [23] and presumably interact with the core particle during virion assembly [24]. The cytoplasmic orientation of the pre-S region is required for the transcriptional activator function of LHB and middle surface protein in vitro $[25,26]$. The luminal orientation of the pre-S domain, after virus maturation and secretion, is exposed on the surface of the virion and is involved in virus attachment and recognition $[27,28]$. Mutants with various types of inframe deletion in pre-S1 region were found to be replication competent in vitro $[17,20,29]$ and can be the predominant strain in vivo [30-32]. In HCC patients, the pre-S mutants are prevalent for up to $63 \%$, including mutants with combinations of deletions over the pre-S1 and pre-S2 regions (Figure 1B) [33,34].

The accumulation of mutant or unfolded proteins causes stress in ER that is sensed by the glucose-regulated protein 78 (Grp78). Unfolded proteins will sequester GRP78 and dissociate from three ER transmembrane transducers leading to their activation [35]. The activation of ER stress has been implicated in a variety of human diseases including neurodegenerative disease, inflammation, and cancer [36]. It has been demonstrated that the secretion of surface proteins was compromised by pre-S deletions, especially pre-S2 mutants [11]. In addition, ectopic expression of pre-S mutant proteins in Huh-7 cells increased the levels of ER chaperones (Grp78 and 94) and activated PERK and C-jun N-terminal kinase (JNK) [11]. These results indicate that both pre-S1 and pre-S2 mutant surface proteins induce ER stress signals in hepatocytes. In consistence with this assumption, elevated Grp78 expression was detected in both type I and type II GGHs in the liver [11]. Northern and Western blot analyses revealed that the pre-S1 mutant induced stronger levels of ER chaperone (Grp78 and 94) response, calcium release, cyclooxygenase-2 (COX-2) and inflammatory cytokines, and oxidative stress intermediates, which tend to result in apoptosis $[11,37,38]$. The pre-S2 mutants, albeit inducing a weaker level of ER stress signal, exhibited higher levels of mutation frequency and transforming capabilities in primary hepatocyte cell line $\mathrm{HH} 4$ [39].

One remarkable biological phenomenon conferred by pre-S1 and pre-S2 mutant proteins is the distinct subcellular localizations of these two proteins in the hepatocytes. Accumulation of pre-S1 mutant proteins frequently displayed an inclusion-like configuration in type I GGHs (Figure 1). In contrast, peripheral or marginal distribution of pre-S2 mutant proteins was evident in type II GGHs $[11,40]$. It is plausible that marginal distribution of pre-S2 mutant proteins is a result of an active recruitment process of pre-S2 mutant proteins, by other ER components, towards the cell periphery. The triggering factor of this recruitment and the patho-biological implication of this process will shed light on the pathogenesis of GGHs as the pre-neoplastic lesions for the development of HCC.

\section{ER stress-dependent and-independent pathways induced by pre-S2 mutants, leading to oxidative DNA damage, genomic instability, and transforming capabilities in transgenic mice model}

The induction of ER stress has been shown to increase the level of reactive oxygen species (ROS), NFkB activation and cyclo-oxygenase-2 (COX-2) expression and thereby induces oxidative DNA damages (Figure 2) [35-37]. Treatments with antioxidants reduced ER stress and improved protein folding [41]. As expected, the expression of pre-S mutant 


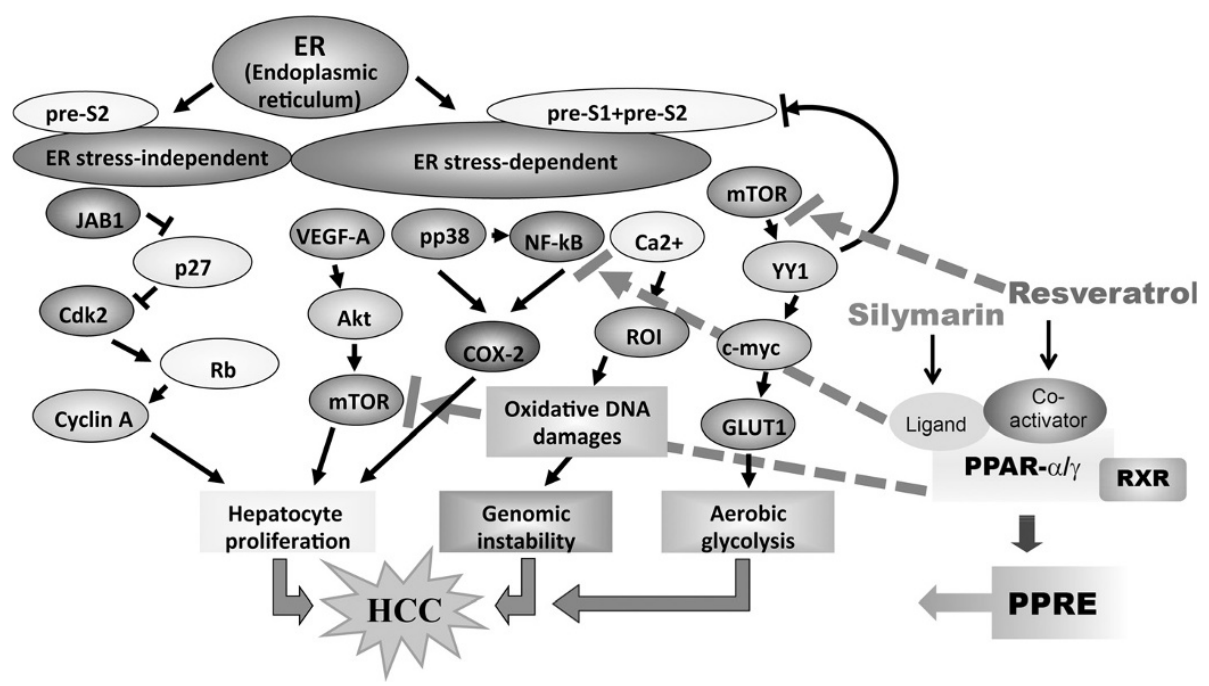

Figure 2 Schematic depiction of the potential signals induced by pre-S mutants and the candidate targets for chemoprevention. Both types of pre-S mutants can induce endoplasmic reticulum (ER) stress signals, which may lead to oxidative stress and DNA damage, leading to genomic instability. The pre-S mutants may also activate two signal pathways to protect the hepatocytes from apoptosis, one involving nuclear factor (NF)-kB to upregulate cyclooxygenase-2 (COX-2) and the other vascular endothelial growth factor to activate Akt/mammalian target of Rapamy- cin (mTOR) signaling. Pre-S2 mutant can additionally induce an ER stress-independent c-Jun activation domain binding protein 1 (JAB1)/ p27/retinoblastoma (Rb)/adenovirus E2 promoter binding factor/cyclin A signal to initiate cell cycle progression. Combined effects of genomic instability and cell proliferation will potentially result in carcinogenesis. Resveratrol and Silymarin are two nature products could be used to target PPAR-a/Y and mTOR signal cascade for chemoprevention in high risk HBV carriers. Cdk2, cyclin-dependent kinase 2; HBV, hepatitis B virus; ROI, reactive oxygen intermediate.

proteins induced oxidative DNA damages, as demonstrated by the increase in 8-hydroxyguanonine on the DNA lesion and increased levels of 8-oxoguanine glycosylase 1 (ogg1) and X-ray cross-complementation 1 (xrcc1) [38]. These studies suggest the presence of genomic instability in GGHs [42]. Notably, as a promising gene transactivator and an ER stress inducer, pre-S2 mutant protein also promotes centrosome instability through two independent mechanisms. First, pre-S2 mutant protein could upregulate cyclin A and sustained cyclin D1 and cyclin-dependent kinase-4 via gene transactivation. This event subsequently promoted cell cycle progression even in the presence of ER stress and resulted in nodular proliferation in transgenic mice livers [39]. Second, ER stress facilitates the release of calcium from the ER and thereby activates calcium-dependent calpain proteases. Notably, cyclin A is a substrate of calpain and the proteolysis results in cytoplasmic redistribution of cyclin A and thereby stimulates centrosome overduplication [43]. These studies demonstrate that pre-S2 mutant protein is a direct driver of genomic instability through the induction of DNA damages and centrosome abnormality.

The most important molecular mechanism initiated by pre-S2 mutant is the VEGF/Akt/mTOR signal pathway which is activated in Huh-7 cells and sequentially activated at the early, middle, and advanced stages of transgenic livers harboring pre-S2 deletion mutant (Figure 2) $[44,45]$. The mTOR signaling is commonly activated in human HCC tissues and represents a candidate target for therapy. The transforming ability of pre-S2 mutant proteins has been investigated in an immortalized human hepatocyte line HH4 [39]. In addition, transgenic mice carrying pre-S2 mutant developed HCC [40]. These studies further support the role of pre-S2 mutants and GGHs in HBV hepatocarcinogenesis $[14,46]$.

Aside from the ER stress-dependent signaling pathways, a distinct ER stress-independent response has been found specifically for pre-S2 deletion mutant protein and is significant for their biologic and carcinogenic preferences [11]. The pre-S2 mutant protein specifically interacts with c-Jun activation domain binding protein 1 (JAB1), which enhances activator protein-1 transcriptional activity and cell proliferation [42]. Through its binding to JAB1, the preS2 mutant protein induces JAB1 nuclear translocation, which activates $\mathrm{p} 27 /$ retinoblastoma/Cdk2/cyclin $\mathrm{A}, \mathrm{D}$ pathways and leads to cell cycle progression and centrosome over-duplication $[39,43]$. These findings have provided clear mechanisms for the growth advantage induced by the preS2 mutant protein.

Two HBV viral proteins, the $\mathrm{X}$ protein $(\mathrm{HBx})$ and pre-S2 deletion mutant protein, have been considered to have either direct or indirect effects in HBV hepatocarcinogenesis [47]. Other than the molecular mechanisms of pre-S2 deletion mutants in hepatocarcinogenesis as mentioned above, $\mathrm{HBx}$ also has been shown to mediate the activation 
of multiple signal pathways including the mTOR signal cascade [48-52]. In our laboratory, we observed a strong $\mathrm{HBx}$ expression in the cytosolic fraction of GGHs in 5 of 20 $\mathrm{HBsAg}$-positive human livers. Interestingly, $\mathrm{HBx}$ was consistently co-expressed with $\mathrm{HBsAg}$, but not vice versa, in GGHs. The expression of both oncoproteins, however, was only rarely detected in HCC tissues [45]. Transgenic mice harboring the $\mathrm{HBx}$, pre-S2 mutant, and a double ( $\mathrm{HBx}$ plus pre-S2 mutant) construct have been established in our laboratory. We observed that the transgenic livers harboring double construct plasmids developed HCCs at an average of 15.1 months, earlier than that of HBx (16.9 months) or preS2 mutant (24.5 months) alone. Interestingly, the oncogenic signals of VEGF-A, p-Akt1/2/3, mitogen-activated protein kinases (MAPK) signaling, and p-mTOR were sequentially and differentially activated at different stages in the progression of tumorigenesis [45]. The combined expression of $\mathrm{HBx}$ and pre-S2 mutant can exhibit enhanced oncogenic effects in HBV tumorigenesis. The exact role of $\mathrm{HBx}$ and pre-S2 mutant protein, either alone or in combination, in human HCC development remains to be clarified.

\section{Presence of pre-S2 mutants in serum predicts the resistance of nucleoside analogues anti-virals and a higher risk of HCC development}

The emergence of pre-S deletion mutants occurs during the natural course of HBV infection, possibly due to selective pressure by the immune system $[32,53]$. The frequencies of pre-S mutation increased successively in different stage of chronic HBV infection. In a metaanalysis study [54], the summarized results showed that pre-S mutants were detected in around $10 \%$ of asymptomatic HBsAg carriers, $20 \%$ of patients with chronic hepatitis B, 35\% of patients with liver cirrhosis and 50\% patients with HCC. The pre-S2 deletion mutants are more frequently detected in anti-HBeAg-positive patients and in patients with $\mathrm{HCC}$ than in $\mathrm{HBeAg}$-positive patients $[32,34,55]$. The ratio of pre-S mutant clones related to wild type in serum also accumulates, as it was $6.4 \%$ at high replicative phase, $13 \%$ at intermediate, and $37.5 \%$ at low or nonreplicative phases [34]. Therefore, pre-S2 mutants represent a significant proportion of virus in advanced stage patients [56,57].

Although anti-viral nucleoside analogues therapy has been associated with a lower risk of HCC development or recurrence after liver resection in chronic HBV carriers [58], the frequencies of pre-S mutants have been reported to be increased after antiviral therapy by nucleos(t)ide analogues which is closely associated with the drug resistance and predict the high risk development of HCC [59,60]. Interestingly, in the control group treated with alpha-interferon, the pre-S2 mutants were significantly reduced or absent, suggesting that alpha-interferon may degrade or inhibit the synthesis of pre-S2 mutant proteins [59]. The presence of
pre-S mutants, especially pre-S2 mutant, has been found to be significantly associated with the risk of HCC development [59-64]. Pre-S deletion mutants detected in serum has also been reported to increase the risk of post-operative recurrence of HCC [65]. Of particular note, pre-S mutation could occur early in age and significantly associated with $\mathrm{HCC}$ in pediatric patients [66,67]. Pre-S2 deletion mutants in sera can be detected in nearly half of children with HCC, in contrast to none in children with chronic HBV infection [66]. By using tissue samples, pre-S2 deletion mutants can be detected in about $80 \%$ of pediatric HCC [67]. Overall, the combined effects of cell cycle progression, genomic instabilities, and survival advantage exhibited by pre-S2 mutant proteins strongly suggest that type II GGHs are potential preneoplastic loci for HCC development and de novo recurrence after surgical resection. In a cohort of 82 patients with HBV-related HCC who received curative surgery [68], type II GGHs were found to be the independent variables associated with late recurrence and the overall survival. However, a prospective cohort study is needed to test the specificity and sensitivity of pre-S2 deletion mutants and ground glass hepatocytes in the predictive value of HCCs.

\section{Development of DNA chip to detect pre-S2 mutants in serum as the predictive hallmark of HCC}

To efficiently detect pre-S deletion mutants in serum, we have successfully developed the oligonucleotide Pre-S Gene Chip to detect the pre-S deletion mutants in sera. The PreS Gene Chip contains 42 DNA probes that target the pre-S region of the LHBS gene, offering a highly sensitive and specific method for pre-S deletion detection with short turnaround time ( $\leq 3$ days) [69]. Screening the pre-S deletion mutants revealed interesting findings that the detection rate of pre-S mutants were relatively low (7\%) in the sera of patients with acute exacerbation of chronic HBV infection but gradually increased in later periods of chronic HBV infection, as they were $37 \%$ in advanced stage of chronic HBV carriers, and as high as 60\% in HCC patients [69]. Combined detection of pre-S mutants and other markers of HBV replication such as HBeAg and viral loads is believed to offer a reliable predictive method for predicting $\mathrm{HCC}$ risks in chronic HBV carriers.

\section{Potential chemoprevention or therapy of HBV-related HCCs targeting at ER stress-induced signal cascade and metabolic disorders}

Recently, metabolic disorders have been shown to be associated with cancer development. In addition to its betterknown functions in promoting protein synthesis and suppressing autophagy, mTOR is now emerging as a key regulator of cellular metabolism and cancer [70]. By analysis of metabolic gene expression profiles in transgenic mice livers and in vitro culture system harboring pre-S2 


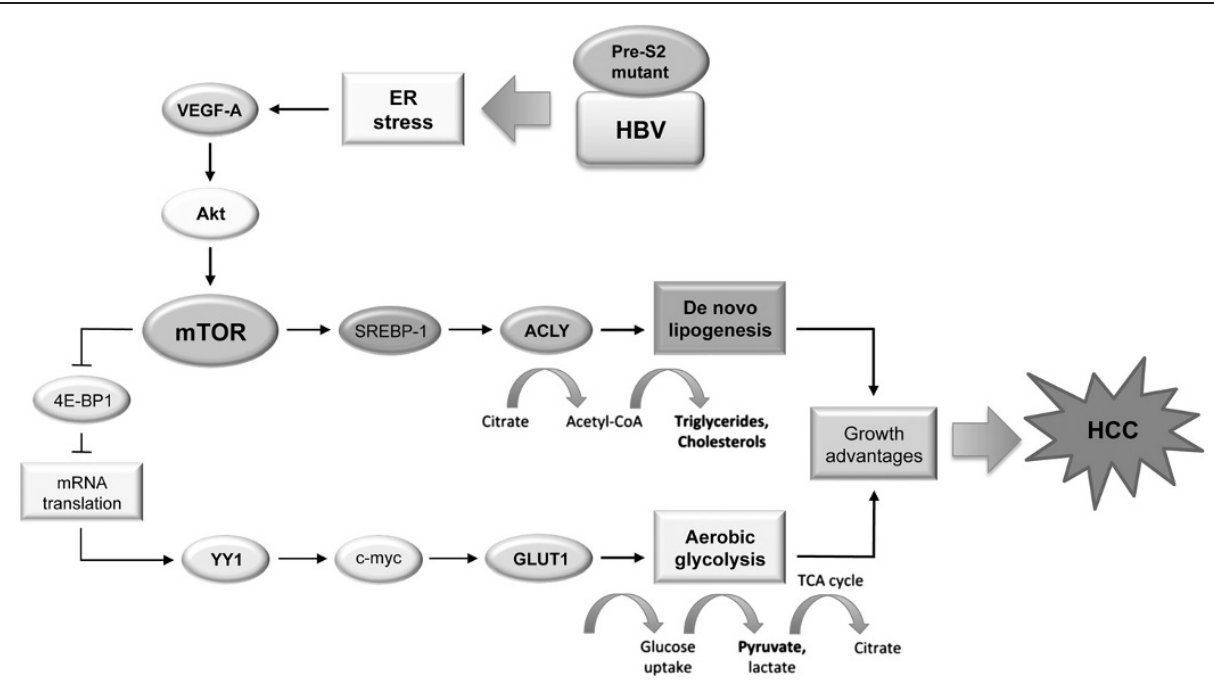

Figure 3 Proposed model of HBV pre-S2 mutant-induced in metabolic disturbance and tumorigenesis. HBV pre-S2 mutant activated mTOR through ER stress-dependent VEGF-A/Akt signal cascade. The activated mTOR induced glycolysis via YY1/c-Myc/Glut1 signaing and promoted de novo lipogenesis through the activation of SREBP-1 and ACLY signals. The combined effects of aerobic glycolysis and de novo lipogenesis contributed to growth advantages of hepatocytes and subsequent HCC development.

mutant protein, we observed that mTOR exhibited a significant role in the metabolic switch toward increased glycolysis and lipid accumulation in HCC tissues (Figure 3). We demonstrated that pre-S2 mutant could activate two mTOR-induced metabolic pathways, one involving Yin Yang 1 (YY1), c-myc and, glucose transporter 1 (GLUT1) to upregulate aerobic glycolysis, and the other involving sterol regulatory element-binding protein-1 (SREBP-1) and acyl-CoA lyase (ACLY) to promote de novo lipid biosynthesis (Teng C.F. et al., unpublished data). The activation of mTOR-dependent metabolic signaling cascades was further validated in human HBV-related HCC tissues. To protect HBV carriers from developing $\mathrm{HCC}$ and preventing recurrence after HCC resection, it is important to develop chemopreventive agents for the high risk patients targeting at the specific signal pathway, with the combination of antivirals. Among the natural products, silymarin and resveratrol may represent potential candidate products because of their popular and long-term usage in human communities [71]. Silymarin, the active component-silibinin, has been evaluated clinically in the treatment of hepatitis and liver damage because of its anti-inflammation and anti-oxidant effects $[71,72]$. Resveratrol has been verified to be effective to prevent cancer development at various stages of carcinogenesis including initiation, promotion, tumor invasion, and metastatsis [73-75]. Importantly, resveratrol is also a promising product for metabolic syndrome mediated through mTOR inhibition and upregulation of PPARs and PGC-1 $\alpha[76,77]$. The combination of silymarin and resveratrol could target on the major signal pathways induced by pre-S2 mutants (Figure 2). Preliminary studies in our laboratory revealed a remarkable effect of this combined product on reducing lipid metabolism and decreasing the incidence of HCC development in transgenic mice harboring HBx and pre-S2 mutant [78]. Further studies or clinical trials are needed to validate the effect of the natural products, with or without the combination of antivirals in chemoprevention or therapy of HBV-related HCCs.

\section{Conclusion}

In this review, we provide a comprehensive overview to provide evidence on the emerging role of HBV pre-S2 deletion mutant protein in HBV tumorigenesis. The HBV pre-S2 deletion mutant proteins are retained in the ER and induce ER stress response. Series of ER stressdependent and -independent growth signals are then activated. Among the diverse pathways, mTOR-mediated signal cascade represent a major mechanism for the disturbed metabolism, genomic instability, and growth advantage, which drive the type II GGHs toward the pre-neoplastic and neoplastic lesions. To identify the patients at high risk for HCC development represents the major task in combating chronic HBV infection in the coming decades. The development of a DNA chip for detecting pre-S2 deletion mutant will meet this demand. Chemopreventive or therapeutic agents can then be provided to these high risk HBV carriers to prevent from HCC development.

\section{Competing interests}

The authors declare that they have no competing interests.

\section{Authors' contributions}

IHS conceived of the study and participated in its design and coordination. LHW carried out the pre-S mutants identification and their signaling pathways. WCH carried out chemoprevention on transgenic mice. HCW carried out 
immunohistochemical staining. CFT identified the impact of pre-S mutants in mTOR signaling cascade regulation. HWT identified GGHs patterns and performed the statistical analysis. WH participated in study design and the development of pre-S DNA chip. All authors contributed to manuscript drafting. All authors read and approved the final manuscript

\section{Acknowledgments}

This study was supported by grants from National Health Research Institutes and National Science Council, Taiwan (Dr. Su and Dr. Huang).

\section{Author details}

${ }^{1}$ National Institute of Infectious Diseases and Vaccinology, National Health Research Institutes, Tainan, Taiwan. 'Department of Pathology, College of Medicine, National Cheng Kung University, Tainan, Taiwan. ${ }^{3}$ The Department of Medical Laboratory Science and Biotechnology, College of Medicine, National Cheng Kung University, Tainan, Taiwan. ${ }^{4}$ Institute of Molecular and Cellular Biology \& Department of Medical Science, National Tsing Hua University, Hsinchu, Taiwan.

Received: 28 April 2014 Accepted: 7 October 2014

Published online: 15 October 2014

\section{References}

1. Beasley RP, Hwang LY, Lin CC, Chien CS: Hepatocellular carcinoma and hepatitis B virus. A prospective study of 22707 men in Taiwan. Lancet 1981, 2:1129-1133.

2. Su IJ, Hsieh WC, Tsai HW, Wu HC: Chemoprevention and novel therapy for hepatocellular carcinoma associated with chronic hepatitis B virus infection. Hepatobiliary Surg Nutr 2013, 2:37-39.

3. Sitia G, Aiolfi R, Di Lucia P, Mainetti M, Fiocchi A, Mingozzi F, Esposito A, Ruggeri ZM, Chisari FV, lannacone M, Guidotti LG: Antiplatelet therapy prevents hepatocellular carcinoma and improves survival in a mouse model of chronic hepatitis B. Proc Natl Acad Sci U S A 2012, 109:E2165-E2172.

4. Liaw YF, Chu CM: Hepatitis B virus infection. Lancet 2009, 373:582-592.

5. Chisari FV, Klopchin K, Moriyama T, Pasquinelli C, Dunsford HA, Sell S, Pinkert CA, Brinster RL, Palmiter RD: Molecular pathogenesis of hepatocellular carcinoma in hepatitis B virus transgenic mice. Cell 1989, 59:1145-1156.

6. Hadziyannis S, Gerber MA, Vissoulis C, Popper H: Cytoplasmic hepatitis B antigen in "ground-glass" hepatocytes of carriers. Arch Pathol 1973, 96:327-330.

7. Popper H: The ground glass hepatocyte as a diagnostic hint. Hum Pathol 1975, 6:517-520.

8. Shikata T: Australia antigen in liver tissue-an immunofluorescent and immunoelectron microscopic study. Jpn J Exp Med 1973, 43:231-245.

9. Gudat F, Bianchi L, Sonnabend W, Thiel G, Aenishaenslin W, Stalder GA: Pattern of core and surface expression in liver tissue reflects state of specific immune response in hepatitis B. Lab Invest 1975, 32:1-9.

10. Hsu HC, Lai MY, Su IJ, Chen DS, Chang MH, Yang PM, Wu CY, Hsieh HC: Correlation of hepatocyte $\mathrm{HBsAg}$ expression with virus replication and liver pathology. Hepatology 1988, 8:749-754.

11. Wang HC, Wu HC, Chen CF, Fausto N, Lei HY, Su IJ: Different types of ground glass hepatocytes in chronic hepatitis B virus infection contain specific pre-S mutants that may induce endoplasmic reticulum stress. Am J Pathol 2003, 163:2441-2449.

12. Su IJ, Kuo TT, Liaw YF: Hepatocyte hepatitis B surface antigen. Diagnostic evaluation of patients with clinically acute hepatitis B surface antigen-positive hepatitis. Arch Pathol Lab Med 1985, 109:400-402.

13. Hsu HC, Lin YH, Chang MH, Su IJ, Chen DS: Pathology of chronic hepatitis $B$ virus infection in children: with special reference to the intrahepatic expression of hepatitis B virus antigens. Hepatology 1988, 8:378-382.

14. Su IJ, Lai MY, Hsu HC, Chen DS, Yang PM, Chuang SM, Sung JL: Diverse virological, histopathological and prognostic implications of seroconversion from hepatitis $\mathrm{B}$ e antigen to anti-HBe in chronic hepatitis B virus infection. J Hepatol 1986, 3:182-189.

15. Fan YF, Lu CC, Chang YC, Chang TT, Lin PW, Lei HY, Su IJ: Identification of a pre-S2 mutant in hepatocytes expressing a novel marginal pattern of surface antigen in advanced diseases of chronic hepatitis $B$ virus infection. J Gastroenterol Hepatol 2000, 15:519-528.
16. Tai PC, Banik D, Lin Gl, Pai S, Pai K, Lin MH, Yuoh G, Che S, Hsu SH, Chen TC, Kuo TT, Lee CS, Yang CS, Shih C: Novel and frequent mutations of hepatitis B virus coincide with a major histocompatibility complex class I-restricted T-cell epitope of the surface antigen. J Virol 1997, 71:4852-4856

17. Tai PC, Suk FM, Gerlich WH, Neurath AR, Shih C: Hypermodification and immune escape of an internally deleted middle-envelope (M) protein of frequent and predominant hepatitis B virus variants. Virology 2002, 292:44-58.

18. Hsu HC, Wu TT, Wu MZ, Wu CY, Chiou TJ, Sheu JC, Lee CS, Chen DS: Evolution of expression of hepatitis B surface and core antigens $(\mathrm{HBs} A g$, $\mathrm{HBcAg}$ ) in resected primary and recurrent hepatocellular carcinoma in HBsAg carriers in Taiwan. Correlation with local host immune response. Cancer 1988, 62:915-921.

19. Melegari M, Scaglioni PP, Wands JR: The small envelope protein is required for secretion of a naturally occurring hepatitis $B$ virus mutant with pre-S1 deleted. J Virol 1997, 71:5449-5454.

20. Xu Z, Yen TS: Intracellular retention of surface protein by a hepatitis B virus mutant that releases virion particles. J Virol 1996, 70:133-140.

21. Bruss V, Lu X, Thomssen R, Gerlich WH: Post-translational alterations in transmembrane topology of the hepatitis $B$ virus large envelope protein. EMBO J 1994, 13:2273-2279.

22. Ostapchuk P, Hearing P, Ganem D: A dramatic shift in the transmembrane topology of a viral envelope glycoprotein accompanies hepatitis $B$ viral morphogenesis. EMBO J 1994, 13:1048-1057.

23. Loffler-Mary H, Werr M, Prange R: Sequence-specific repression of cotranslational translocation of the hepatitis B virus envelope proteins coincides with binding of heat shock protein Hsc70. Virology 1997, 235:144-152.

24. Le Pogam S, Shih C: Influence of a putative intermolecular interaction between core and the pre-S1 domain of the large envelope protein on hepatitis B virus secretion. J Virol 2002, 76:6510-6517.

25. Hildt E, Hofschneider PH: The PreS2 activators of the hepatitis B virus: activators of tumour promoter pathways. Recent Results Cancer Res 1998, 154:315-329.

26. Hildt E, Urban S, Eckerskorn C, Hofschneider PH: Isolation of highly purified, functional carboxy-terminally truncated hepatitis $B$ virus middle surface protein activators from eucaryotic expression systems. Hepatology 1996, 24:502-507.

27. Klingmuller $\mathrm{U}$, Schaller $\mathrm{H}$ : Hepadnavirus infection requires interaction between the viral pre-S domain and a specific hepatocellular receptor. J Virol 1993, 67:7414-7422

28. Neurath AR, Kent SB, Strick N, Parker K: Identification and chemical synthesis of a host cell receptor binding site on hepatitis $B$ virus. Cell 1986, 46:429-436.

29. Melegari M, Bruno S, Wands JR: Properties of hepatitis B virus pre-S1 deletion mutants. Virology 1994, 199:292-300.

30. Bock CT, Tillmann HL, Maschek HJ, Manns MP, Trautwein C: A preS mutation isolated from a patient with chronic hepatitis B infection leads to virus retention and misassembly. Gastroenterology 1997, 113:1976-1982.

31. Pult I, Chouard T, Wieland S, Klemenz R, Yaniv M, Blum HE: A hepatitis B virus mutant with a new hepatocyte nuclear factor 1 binding site emerging in transplant-transmitted fulminant hepatitis B. Hepatology 1997, 25:1507-1515.

32. Santantonio $T$, Jung MC, Schneider R, Fernholz D, Milella M, Monno L, Pastore G, Pape GR, Will H: Hepatitis B virus genomes that cannot synthesize pre-S2 proteins occur frequently and as dominant virus populations in chronic carriers in Italy. Virology 1992, 188:948-952.

33. Su IJ, Wang HC, Wu HC, Huang WY: Ground glass hepatocytes contain pre-S mutants and represent preneoplastic lesions in chronic hepatitis B virus infection. J Gastroenterol Hepatol 2008, 23:1169-1174.

34. Fan YF, Lu CC, Chen WC, Yao WJ, Wang HC, Chang TT, Lei HY, Shiau AL, Su $\mathrm{IJ}$ : Prevalence and significance of hepatitis B virus (HBV) pre-S mutants in serum and liver at different replicative stages of chronic HBV infection. Hepatology 2001, 33:277-286

35. Malhi H, Kaufman RJ: Endoplasmic reticulum stress in liver disease. J Hepatol 2011, 54:795-809.

36. Wang S, Kaufman RJ: The impact of the unfolded protein response on human disease. J Cell Biol 2012, 197:857-867.

37. Hung JH, Su IJ, Lei HY, Wang HC, Lin WC, Chang WT, Huang W, Chang WC, Chang YS, Chen CC, Lai MD: Endoplasmic reticulum stress stimulates the expression of cyclooxygenase-2 through activation of NF-kappaB and pp 38 mitogen-activated protein kinase. J Biol Chem 2004, 279:46384-46392. 
38. Hsieh YH, Su IJ, Wang HC, Chang WW, Lei HY, Lai MD, Chang WT, Huang W: Pre-S mutant surface antigens in chronic hepatitis $B$ virus infection induce oxidative stress and DNA damage. Carcinogenesis 2004, 25:2023-2032.

39. Wang HC, Chang WT, Chang WW, Wu HC, Huang W, Lei HY, Lai MD, Fausto $N$, Su IJ: Hepatitis B virus pre-S2 mutant upregulates cyclin A expression and induces nodular proliferation of hepatocytes. Hepatology 2005, 41:761-770.

40. Wang HC, Huang W, Lai MD, Su IJ: Hepatitis B virus pre-S mutants, endoplasmic reticulum stress and hepatocarcinogenesis. Cancer Sci 2006. 97:683-688.

41. Malhotra JD, Miao H, Zhang K, Wolfson A, Pennathur S, Pipe SW, Kaufman $\mathrm{RJ}$ : Antioxidants reduce endoplasmic reticulum stress and improve protein secretion. Proc Natl Acad Sci U S A 2008, 105:18525-18530.

42. Hsieh $\mathrm{YH}, \mathrm{Hsu} J \mathrm{~L}, \mathrm{Su} \mathrm{IJ}$, Huang W: Genomic instability caused by hepatitis B virus: into the hepatoma inferno. Front Biosci (Landmark Ed) 2011, $16: 2586-2597$

43. Wang LH, Huang W, Lai MD, Su IJ: Aberrant cyclin A expression and centrosome overduplication induced by hepatitis B virus pre-S2 mutants and its implication in hepatocarcinogenesis. Carcinogenesis 2012, 33:466-472.

44. Yang JC, Teng CF, Wu HC, Tsai HW, Chuang HC, Tsai TF, Hsu YH, Huang W, Wu LW, Su IJ: Enhanced expression of vascular endothelial growth factor-A in ground glass hepatocytes and its implication in hepatitis B virus hepatocarcinogenesis. Hepatology 2009, 49:1962-1971.

45. Wu HC, Tsai HW, Teng CF, Hsieh WC, Lin YJ, Wang LHC, Yuan Q, Su IJ: Ground-glass hepatocytes co-expressing hepatitis B virus $X$ protein and surface antigens exhibit enhanced oncogenic effects and tumorigenesis. Hum Pathol 2014, 45:1294-1301.

46. Mathai AM, Alexander J, Kuo FY, Torbenson M, Swanson PE, Yeh MM: Type II ground-glass hepatocytes as a marker of hepatocellular carcinoma in chronic hepatitis B. Hum Pathol 2013, 44:1665-1671.

47. Tan A, Yeh SH, Liu CJ, Cheung C, Chen PJ: Viral hepatocarcinogenesis: from infection to cancer. Liver Int 2008, 28:175-188.

48. Benn J, Schneider RJ: Hepatitis B virus HBx protein activates Ras-GTP complex formation and establishes a Ras, Raf, MAP kinase signaling cascade. Proc Natl Acad Sci U S A 1994, 91:10350-10354.

49. Chan CF, Yau TO, Jin DY, Wong CM, Fan ST, Ng IO: Evaluation of nuclear factor-kappaB, urokinase-type plasminogen activator, and $\mathrm{HBx}$ and their clinicopathological significance in hepatocellular carcinoma. Clin Cancer Res 2004, 10:4140-4149.

50. Lee $\mathrm{Yl}$, Kang-Park S, Do SI, Lee YI: The hepatitis B virus-X protein activates a phosphatidylinositol 3-kinase-dependent survival signaling cascade. J Biol Chem 2001, 276:16969-16977.

51. Lee $Y H$, Yun Y: HBx protein of hepatitis B virus activates Jak1-STAT signaling. J Biol Chem 1998, 273:25510-25515.

52. Yen CJ, Lin YJ, Yen CS, Tsai HW, Tsai TF, Chang KY, Huang WC, Lin PW, Chiang CW, Chang TT: Hepatitis B virus $X$ protein upregulates mTOR signaling through IKKbeta to increase cell proliferation and VEGF production in hepatocellular carcinoma. PLoS One 2012, 7:e41931.

53. Mimms L: Hepatitis B virus escape mutants: "pushing the envelope" of chronic hepatitis B virus infection. Hepatology 1995, 21:884-887.

54. Liu S, Zhang H, Gu C, Yin J, He Y, Xie J, Cao G: Associations between hepatitis $B$ virus mutations and the risk of hepatocellular carcinoma: a meta-analysis. J Nat/ Cancer Inst 2009, 101:1066-1082.

55. Huy $\Pi$, Ushijima $H$, Win KM, Luengrojanakul P, Shrestha PK, Zhong ZH, Smirnov AV, Taltavull TC, Sata T, Abe K: High prevalence of hepatitis B virus pre-s mutant in countries where it is endemic and its relationship with genotype and chronicity. J Clin Microbiol 2003, 41:5449-5455.

56. Fernholz D, Galle PR, Stemler M, Brunetto M, Bonino F, Will H: Infectious hepatitis $B$ virus variant defective in pre-S2 protein expression in a chronic carrier. Virology 1993, 194:137-148.

57. Le Seyec J, Chouteau P, Cannie I, Guguen-Guillouzo C, Gripon P: Role of the pre-S2 domain of the large envelope protein in hepatitis $B$ virus assembly and infectivity. J Virol 1998, 72:5573-5578.

58. Wu CY, Chen YJ, Ho HJ, Hsu YC, Kuo KN, Wu MS, Lin JT: Association between nucleoside analogues and risk of hepatitis $B$ virus-related hepatocellular carcinoma recurrence following liver resection. JAMA 2012, 308:1906-1914.

59. Zhang D, Dong P, Zhang K, Deng L, Bach C, Chen W, Li F, Protzer U, Ding $H$, Zeng $C$ : Whole genome HBV deletion profiles and the accumulation of preS deletion mutant during antiviral treatment. BMC Microbiol 2012, 12:307.

60. Pollicino T, Cacciola I, Saffioti F, Raimondo G: Hepatitis B virus PreS/S gene variants: Pathobiology and clinical implications. J Hepatol 2014, 61:408-417.
61. Kao JH, Liu CJ, Jow GM, Chen PJ, Chen DS, Chen BF: Fine mapping of hepatitis $B$ virus pre-S deletion and its association with hepatocellular carcinoma. Liver Int 2012, 32:1373-1381.

62. Fang ZL, Sabin CA, Dong BQ, Wei SC, Chen QY, Fang KX, Yang JY, Huang J, Wang $X Y$, Harrison TJ: Hepatitis $B$ virus pre-S deletion mutations are a risk factor for hepatocellular carcinoma: a matched nested case-control study. J Gen Virol 2008, 89:2882-2890.

63. Sinn DH, Choi MS, Gwak GY, Paik YH, Lee JH, Koh KC, Paik SW, Yoo BC: Pre-s mutation is a significant risk factor for hepatocellular carcinoma development: a long-term retrospective cohort study. Dig Dis Sci 2013, 58:751-758.

64. Chen $\mathrm{CH}$, Hung $\mathrm{CH}$, Lee $\mathrm{CM}$, Hu TH, Wang JH, Wang JC, Lu SN, Changchien CS: Pre-S deletion and complex mutations of hepatitis $B$ virus related to advanced liver disease in HBeAg-negative patients. Gastroenterology 2007, 133:1466-1474.

65. Su CW, Chiou YW, Tsai YH, Teng RD, Chau GY, Lei HJ, Hung HH, Huo TI, Wu $J C$ : The influence of hepatitis B viral load and pre-S deletion mutations on post-operative recurrence of hepatocellular carcinoma and the tertiary preventive effects by anti-viral therapy. PLoS One 2013, 8:e66457.

66. Huang HP, Hsu HY, Chen CL, Ni YH, Wang HY, Tsuei DJ, Chiang CL, Tsai YC, Chen $\mathrm{HL}$, Chang $\mathrm{MH}$ : Pre-S2 deletions of hepatitis $\mathrm{B}$ virus and hepatocellular carcinoma in children. Pediatr Res 2010, 67:90-94

67. Abe K, Thung SN, Wu HC, Tran TT, Le Hoang P, Truong KD, Inui A, Jang J J, Su IJ: Pre-S2 deletion mutants of hepatitis B virus could have an important role in hepatocarcinogenesis in Asian children. Cancer Sci 2009, 100:2249-2254.

68. Tsai HW, Lin YJ, Lin PW, Wu HC, Hsu KH, Yen CJ, Chan SH, Huang W, Su IJ: A clustered ground-glass hepatocyte pattern represents a new prognostic marker for the recurrence of hepatocellular carcinoma after surgery. Cancer 2011, 117:2951-2960.

69. Shen FC, Su IJ, Wu HC, Hsieh YH, Yao WJ, Young KC, Chang TC, Hsieh HC, Tsai $\mathrm{HN}$, Huang W: A pre-S gene chip to detect pre-S deletions in hepatitis $B$ virus large surface antigen as a predictive marker for hepatoma risk in chronic hepatitis B virus carriers. J Biomed Sci 2009, 16:84

70. Cornu M, Albert V, Hall MN: mTOR in aging, metabolism, and cancer. Curr Opin Genet Dev 2013, 23:53-62.

71. Loguercio C, Andreone P, Brisc C, Brisc MC, Bugianesi E, Chiaramonte M, Cursaro C, Danila M, de Sio I, Floreani A, Freni MA, Grieco A, Groppo M, Lazzari R, Lobello S, Lorefice E, Margotti M, Miele L, Milani S, Okolicsanyi L, Palasciano G, Portincasa P, Saltarelli P, Smedile A, Somalvico F, Spadaro A, Sporea I, Sorrentino P, Vecchione R, Tuccillo C, et al: Silybin combined with phosphatidylcholine and vitamin $\mathrm{E}$ in patients with nonalcoholic fatty liver disease: a randomized controlled trial. Free Radic Biol Med 2012, 52:1658-1665.

72. Polyak SJ, Morishima C, Lohmann V, Pal S, Lee DY, Liu Y, Graf TN, Oberlies $\mathrm{NH}$ : Identification of hepatoprotective flavonolignans from silymarin. Proc Natl Acad Sci U S A 2010, 107:5995-5999.

73. Jang M, Cai L, Udeani GO, Slowing KV, Thomas CF, Beecher CW, Fong HH, Farnsworth NR, Kinghorn AD, Mehta RG, Moon RC, Pezzuto JM: Cancer chemopreventive activity of resveratrol, a natural product derived from grapes. Science 1997, 275:218-220.

74. Gusman J, Malonne H, Atassi G: A reappraisal of the potential chemopreventive and chemotherapeutic properties of resveratrol. Carcinogenesis 2001, 22:1111-1117.

75. Fulda S: Resveratrol and derivatives for the prevention and treatment of cancer. Drug Discov Today 2010, 15:757-765.

76. Guarente L: Sirtuins as potential targets for metabolic syndrome. Nature 2006, 444:868-874.

77. Lekli I, Ray D, Das DK: Longevity nutrients resveratrol, wines and grapes. Genes Nutr 2010, 5:55-60

78. Hsieh WC, Yang CW, Haung YS, Chao TW, Tsai TF, Su IJ: Chemoprevention of HBV-related hepatocellular carcinoma by the combined product of resveratrol and silymarin in transgenic mice. Funct Foods Health Dis 2013, 3:341-352.

doi:10.1186/s12929-014-0098-7

Cite this article as: Su et al:: The emerging role of hepatitis B virus Pre$\mathrm{S} 2$ deletion mutant proteins in HBV tumorigenesis. Journal of Biomedical Science 2014 21:98 